\title{
Isotermas de dessorção de filé de bonito (Sarda sarda) desidratado osmoticamente e defumado
}

\author{
Míriam D. Hubinger ${ }^{1}$, David Vivanco-Pezantes ${ }^{1}$, Louise E. Kurozawaa ${ }^{1}$ \& Paulo J. A. Sobral ${ }^{2}$
}

\section{RESUMO}

O presente trabalho teve como objetivo a obtenção de isotermas de dessorção de filés de bonito (Sarda sarda), previamente salgados a vácuo e defumados com fumaça líquida. As isotermas foram obtidas a quatro temperaturas $(5,25,40$ e $60{ }^{\circ} \mathrm{C}$ ) em condições de dessorção, através do método gravimétrico estático, com soluções salinas saturadas. Os dados experimentais foram ajustados a quatro modelos da literatura (BET linearizado, GAB, Henderson e Oswin modificado). Os resultados mostraram que as isotermas tomaram forma sigmoidal de tipo II e que o modelo de Guggenheim-Anderson-deBoer (GAB) foi aceitável para modelar os dados experimentais. O calor isostérico de dessorção, um parâmetro necessário para simular e projetar adequadamente o secador, também foi calculado e pode ser representado através de um modelo matemático simples, em função da umidade de equilíbrio.

Palavras-chave: salga, desidratação, defumação

\section{Desorption isotherms of osmotically dehydrated and smoked Atlantic bonito (Sarda sarda) fillets}

\begin{abstract}
The main objetive of this work was to obtain the desorption isotherms of Atlantic bonito (Sarda sarda) fillets, previously subjected to an immersion impregnation process with $\mathrm{NaCl}$ solutions and smoked in liquid smoke. The isotherms were obtained at four temperatures $\left(5,25,40\right.$ and $\left.60^{\circ} \mathrm{C}\right)$, using the static method with saturated salt solutions. The experimental data were fitted to four models (linearized BET, GAB, Henderson and modified Oswin). The results showed that a type II sigmoidal isotherm, with GAB equation, gave the best fit. The isosteric heat of desorption was also calculated and a simple empirical equation has been proposed to represent this heat, as a function of equilibrium moisture content.
\end{abstract}

Key words: salting, dehydration, smoking

1 FEAGRI/UNICAMP, CEP 13081-970, Campinas, SP. Fone: (19) 3521-4036, E-mail: mhub@fea.unicamp.br, dvivancopezantes@yahoo.com, louise@fea.unicamp.br 2 FZEA/USP, CEP 13630-970 Pirassununga, SP. Fone: (19) 3565-4192. E-mail: pjsobral@usp.br 


\section{INTRODUÇÃO}

O bonito é um peixe marinho de ocorrência no litoral brasileiro, comercializado fresco, congelado e/ou enlatado; representa uma potencial matéria-prima para a indústria pesqueira além de valores energéticos e conteúdo de proteínas, vitaminas e minerais importantes em relação a outras fontes alimentícias.

A salga, secagem e a defumação, constituem técnicas combinadas e tradicionais de conservação de alimentos praticadas pelo homem, as quais têm sido utilizadas em pescado por serem relativamente simples, de baixo custo e garantirem a estabilidade do produto, inclusive a temperatura ambiente. A salga de produtos cárneos ou a base de peixe, pode alterar, de maneira positiva, certas características sensoriais do produto, como o sabor, a textura e a cor (Sabadini et al., 1998; Doe, 1998; Chiralt et al., 2001).

As isotermas de sorção de umidade são característica fundamental dos materiais alimentícios, essenciais para o processo de secagem e relacionadas a quase todos os aspectos de estabilidade à estocagem, de produtos secos ou de produtos a baixa umidade. O comportamento das isotermas de sorção é ainda necessário para se conhecer bem a sua relação com as características físicas, químicas e de estabilidade dos produtos desidratados ou parcialmente desidratados.

Dados de isotermas de sorção de produtos de peixe ou mesmo de pescado desidratado, são escassos na literatura, embora o consumo de pescado, salgado e seco, seja bastante disseminado, sobretudo nos países em desenvolvimento que encontram, nesse tipo de produto, abundante fonte de proteína. Ariahu et al. (2006) apresentam as isotermas de um crustáceo muito comum em águas da Nigéria, cujo nome científico é Procambarus clarkii, altamente perecível e bastante consumido na forma desidratada, naquele país. As isotermas foram construídas pelo método gravimétrico, nas temperaturas de $25,30,40$ e $50^{\circ} \mathrm{C}$, em que os resultados são analisados de acordo com vários modelos de ajuste de isotermas, disponíveis na literatura e os calores de sorção calculados. As equações de GAB, Henderson e Oswin, foram as que apresentaram os melhores ajustes aos dados experimentais. Sablani et al. (2001), ao analisarem o comportamento de isotermas de sorção a temperaturas de 25,40 e $50{ }^{\circ} \mathrm{C}$, de sardinhas liofilizadas usando o método gravimétrico, concluíram que o modelo de GAB foi capaz de predizer os conteúdos de umidade de equilíbrio com um erro menor que $10 \%$ e um bom coeficiente de correlação. O calor isostérico de sorção para este produto, nessas condições, também foi determinado.

As isotermas de sorção da carne de tambaqui (Colossoma macroparum), desidratada osmoticamente, foram determinadas a três temperaturas $\left(5,17\right.$ e $\left.29^{\circ} \mathrm{C}\right)$ e com duas concentrações de solução osmótica (10 e 30\% de $\mathrm{NaCl}$ ), pelo método gravimétrico, por Molina Filho et al. (2006). Quatro modelos de sorção foram testados para verificar o melhor ajuste: GAB, Oswin, BET e Peleg. Os dados experimentais se ajustaram satisfatoriamente aos modelos, sendo o de Peleg o que melhor se correlacionou com os dados experimentais. A influência do teor de $\mathrm{NaCl}$ no comportamento termodinâmico dos filés de tambaqui também foi avaliada pelos autores.
A determinação do calor isostérico de sorção (também chamado calor latente de vaporização), $\mathrm{Q}_{\mathrm{st}}$, em alimentos, é de interesse prático para a realização da etapa de secagem; este valor não é constante nem igual ao calor latente de vaporização da água pura, mas é uma função do conteúdo de umidade e da temperatura, variando ao longo do processo de secagem (Rucklod et al., 2003). A determinação deste calor é feita através das isotermas de sorção, pelo uso de modelos matemáticos simples (Chen, 2006).

Os dados da isoterma de dessorção de filés de peixe previamente desidratados em solução de salmoura por impregnação-imersão e posteriormente defumados, podem ajudar a entender a natureza das mudanças sofridas pelo produto durante a secagem; assim, os objetivos deste trabalho foram: obter as isotermas de dessorção do peixe bonito, osmoticamente desidratado a uma concentração de salmoura (15\% $\mathrm{NaCl}$ ) e defumado, e analisar a influência de quatro temperaturas $\left(5,25,40\right.$ e $\left.60^{\circ} \mathrm{C}\right)$ sobre o comportamento da curva de dessorção do produto.

\section{MATERIAL E MÉTODOS}

\section{Preparo das amostras}

O pescado da espécie bonito, procedente do porto de Santos, foi comprado no Mercado Municipal de Campinas, de fornecedor contratado para obtê-lo. Os pescados foram mantidos em gelo imediatamente após a captura e transportados para o local do experimento. Para o preparo da matéria-prima as vísceras foram eliminadas manualmente, através de um corte na região abdominal, desde a cauda até a cabeça, e se realizaram os cortes para a obtenção de filés com pele, que foram lavados com água destilada, acondicionados em filmes plásticos e resfriados a $1{ }^{\circ} \mathrm{C}$. As amostras resfriadas foram cortadas em dimensões de $7 \mathrm{~cm}$ de comprimento, $5 \mathrm{~cm}$ de largura e $1 \mathrm{~cm}$ de espessura, com peso médio de 45 g; sua caracterização química foi realizada de acordo com procedimento descrito em AOAC (1997).

\section{Desidratação osmótica sob pulso de vácuo}

Para preparo da solução osmótica usou-se água destilada e sal para consumo humano. Para este estudo, a concentração utilizada foi de $15 \%\left(\mathrm{p} \mathrm{p}^{-1}\right)$ de cloreto de sódio. Previamente ao processo de desidratação osmótica as amostras de filé de bonito foram pesadas e identificadas e, a seguir, acondicionadas em bandejas e depois inseridas no equipamento desidratador a vácuo, com capacidade para 22 L de solução, cujas operação e descrição se encontram detalhadas em Vivanco-Pezantes (2006). Os testes foram conduzidos nas condições de temperatura e concentração da salmoura de $5{ }^{\circ} \mathrm{C}$ e $15 \%$, respectivamente; a solução osmótica dentro do tanque recirculava a uma vazão de $2,5 \mathrm{~m}^{3} \mathrm{~h}^{-1}$. A aplicação de uma pressão de vácuo inicial de $150 \mathrm{mbar}$ era feita durante os primeiros $10 \mathrm{~min}$, continuando o processo a pressão atmosférica, até completar $1 \mathrm{~h}$; depois da desidratação as amostras dos filés eram drenadas e colocadas sobre papel absorvente, para a remoção de solução em excesso, e só então pesadas. 


\section{Defumação}

Os filés foram defumados mediante um sistema de bicos aspersores, durante 30 segundos; a fumaça líquida usada para este processo, foi adquirida da Adicon Indústria e Comércio de Aditivos Ltda. (São Bernardo do Campo, SP), com as seguintes características: coloração marrom escuro; odor característico de defumado; densidade: $1.080-1.160 \mathrm{~kg} \mathrm{~m}^{-3}$; compostos aromáticos: 15,0-22,0 $\mathrm{mg} \mathrm{mL}^{-1}$, acidez total, como ácido acético 13,0-16,0\%. Maiores detalhes sobre a descrição dos bicos utilizados e do procedimento experimental de defumação, podem ser encontrados em Vivanco-Pezantes (2006).

\section{Isotermas de dessorção}

Obtiveram-se amostras de filé de peixe salgadas, defumadas e sem pele, usando-se aproximadamente $4,5 \mathrm{~g}$ de músculo de pescado cortado em forma de paralelepípedos de 3 x 4 x 5 mm (com o intuito de melhorar o fenômeno de transferência de massa). A construção das isotermas foi realizada através da técnica gravimétrica, mediante o método estático, com registro descontínuo da variação de peso. Dez sais foram selecionados ( $\mathrm{LiCl}, \mathrm{CH}_{3} \mathrm{COOK}, \mathrm{MgCl}_{2}$, $\mathrm{K}_{2} \mathrm{CO}_{3}$, $\left(\mathrm{NO}_{3}\right)_{2} \mathrm{Mg}, \mathrm{NaBr}, \mathrm{NaCl}, \mathrm{KI}, \mathrm{KCl}, \mathrm{BaCl}_{2}$ ), promovendo ambientes com diferentes umidades relativas na faixa de 10 a 90\% em seu interior (Greenspan, 1977; Kitic et al., 1986). As soluções foram distribuídas em potes individuais com capacidade para apenas uma amostra, em que se utilizaram três potes para cada solução (amostras em triplicata); em cada pote foram colocados aproximadamente $40 \mathrm{~mL}$ de solução salina saturada e um tripé de acrílico devidamente limpo e, sobre este, o recipiente plástico com as amostras; a seguir, foi fechado com tampa hermética. $\mathrm{O}$ pote de plástico e o tripé foram limpos com algodão embebido em formol (comercial a 40\%), a fim de evitar contaminações da amostra; os potes foram acondicionados em estufa com circulação de ar, a temperaturas de 5, 25, 40 e $60{ }^{\circ} \mathrm{C}$; pesagens periódicas das cápsulas contendo as amostras, eram realizadas em balança analítica (marca Sartorius A200S $\pm 0,0001 \mathrm{~g}$ ), a cada 4 dias, até que a massa se tornou constante, depois de aproximadamente 18 a 25 dias, ponto em que se pode dizer que a amostra atingiu o equilíbrio determinando-se, então, a umidade final (em base seca) da amostra pelo método de estufa obtendo-se então os valores de umidade de equilíbrio, que eram correlacionados com a umidade relativa ou atividade de água.

\section{Ajuste dos modelos das isotermas}

Para determinação dos parâmetros dos modelos das isotermas usou-se o método de regressão não linear LevenbergMarquardt do programa Statistica 6.0, com o número máximo de iterações de 100 e um critério de convergência de $1 \times 10^{-5}$. Com o objetivo de se escolher um modelo para a determinação das isotermas de dessorção de filés de Bonito, utilizaram-se quatro modelos propostos na literatura para os respectivos ajustes dos dados experimentais; enfim, os critérios usados para a escolha do melhor ajuste foram os coeficientes de determinação $\left(\mathrm{R}^{2}\right)$ e o valor do erro relativo médio (MRE) definido como:

$$
\text { MRE }=\frac{100}{\mathrm{~N}} \sum_{\mathrm{i}=1}^{\mathrm{N}} \frac{\left|\mathrm{V}_{\mathrm{P}}-\mathrm{V}_{0}\right|}{\mathrm{V}_{0}}
$$

donde $V_{f}$ e $V_{0}$ representam os valores preditos e experimentais da umidade de equilíbrio e $\mathrm{N}$, o número total de pontos. Os melhores ajustes são os que apresentam maior $\mathrm{R}^{2}$ (próximo à unidade) e menor MRE (próximo a 10\%).

\section{Modelos:}

Isoterma de BET. Este modelo foi proposto por BET (1938):

$$
\frac{a_{w}}{\left(1-a_{w}\right) \cdot X_{e}}=\frac{1}{X_{m} \cdot C}+\frac{a_{w} \cdot(C-1)}{X_{m} \cdot C}
$$

em que:

$\mathrm{X}_{\mathrm{e}}$ - umidade de equilíbrio do produto, Kg água (Kg sólido seco) ${ }^{-1}$

$\mathrm{X}_{\mathrm{m}}$ - conteúdo de umidade da monocamada

$\mathrm{a}_{\mathrm{w}}$ - atividade de água, $\mathrm{g} \mathrm{g}^{-1}$

$\mathrm{C}$ - constante relacionada ao excesso de entalpia de sorção

Isoterma de GAB. Este modelo considera três parâmetros, relacionados da seguinte forma (Anderson, 1946; Saravacos et al., 1986)

$$
\frac{X_{e}}{X_{m}}=\frac{C \cdot K \cdot a_{w}}{\left[\left(1-K \cdot a_{w}\right)\left(1+(C-1) K \cdot a_{w}\right)\right]}
$$

em que:

$\mathrm{X}_{\mathrm{e}}$ - umidade de equilíbrio do produto, kg água ( $\mathrm{kg}$ sólido seco) $)^{-1}$

$\mathrm{X}_{\mathrm{m}}$ - umidade do produto, correspondente à situação na qual os pontos primários de adsorção estão saturados por moléculas de água (conceito equivalente à camada monomolecular de BET)

$\mathrm{a}_{\mathrm{w}}$ - atividade de água

C e K - são as constantes de adsorção relacionadas com as interações energéticas entre as moléculas da monocamada e as subseqüentes, em dado sítio de sorção característica do produto.

$$
\mathrm{C}=\frac{\mathrm{N} \exp \left(\mathrm{Q}_{1}-\mathrm{H}_{\mathrm{v}}\right)}{\mathrm{RT}}
$$

em que:

$$
\begin{aligned}
& \mathrm{Q}_{1} \text { - calor de vaporização da água na primeira ca- } \\
& \quad \text { mada monomolecular } \\
& \mathrm{H}_{\mathrm{V}} \text { - calor de vaporização } \\
& \mathrm{R} \text { - constantes dos gases } \\
& \mathrm{T} \text { - temperatura absoluta } \\
& \mathrm{N} \text { - parâmetro característico do produto }
\end{aligned}
$$

Os idealizadores do modelo estenderam as teorias de adsorção física de Langmuir e BET, resultando em uma equação de três parâmetros, conhecida como equação de GAB, matematicamente adequada para aplicações em engenharia e que permite um ótimo ajuste de dados de sorção de quase 
todos os alimentos, na faixa de atividade de água entre 01 a 0,9; apresenta as seguintes vantagens: tem uma base teórica fundamentada, é uma equação matemática de três parâmetros relativamente simples, que têm significado físico, sendo capaz de descrever algum efeito da temperatura, no processo de sorção.

Isoterma de Henderson. Este modelo considera fundamentos termodinâmicos, inclui a temperatura e foi proposto por Henderson (1952).

$$
1-\mathrm{a}_{\mathrm{w}}=\exp \left[-\mathrm{C}(273+\mathrm{t}) \cdot \mathrm{X}_{\mathrm{e}}^{\mathrm{n}}\right]
$$

em que:

$$
\begin{aligned}
\mathrm{C} \text { e } \mathrm{n} \text { - } & \text { são as constantes características do material } \\
\mathrm{t}- & \text { temperatura do sistema em equilíbrio, }{ }^{\circ} \mathrm{C} \\
\mathrm{X}_{\mathrm{e}}- & \text { umidade de equilíbrio do produto, } \mathrm{kg} \text { água } \\
& \left(\mathrm{kg} \text { sólido seco) }{ }^{-1}\right. \\
\mathrm{a}_{\mathrm{w}}- & \text { atividade de água }
\end{aligned}
$$

A equação de Henderson tem sido bastante usada no campo da tecnologia de alimentos reproduzindo bem as isotermas de equilíbrio de alimentos em geral, para valores de atividade de água compreendidos entre 0,10 e 0,71 (Vidal, 1983).

Isoterma de Oswin - modificada. Este modelo matemático considera o efeito da temperatura entre a dependência do conteúdo da umidade de equilíbrio e a atividade de água do produto (Veltchev \& Menkov, 2000):

$$
X_{e}=(A+B \cdot t) \cdot\left(\frac{a_{w}}{1-a_{w}}\right)^{C}
$$

em que:
A, B e C - coeficientes do modelo
$\mathrm{X}_{\mathrm{e}}$ - umidade de equilíbrio do produto, kg água (kg sólido seco) ${ }^{-1}$
$\mathrm{a}_{\mathrm{w}}$ - atividade de água
$\mathrm{t}$ - temperatura do sistema em equilíbrio, ${ }^{\circ} \mathrm{C}$

\section{Calor isostérico de sorção}

O calor isostérico de sorção $\mathrm{Q}_{\mathrm{st}}\left(\mathrm{kJ} \mathrm{kg}^{-1}\right)$ foi determinado pela equação de Clausius-Clapeyron (Labuza et al., 1985; Kechaou \& Maalej, 1999):

$$
\Delta \mathrm{Q}_{\mathrm{st}}=-\mathrm{R}\left(\frac{\partial\left(\ln \mathrm{a}_{\mathrm{w}}\right)}{\partial(1 / \mathrm{T})}\right)
$$

donde $\mathrm{R}$ é a constante universal dos gases; $\mathrm{a}_{\mathrm{w}}$ é a atividade de água e T é a temperatura absoluta, em K.

\section{RESULTADOS E DISCUSSÃO}

A caracterização físico-química dos filés de bonito, fresco e desidratado osmoticamante em solução de salmoura e defumado, mostrada na Tabela 1 , revela que os dois produtos, embora semelhantes entre si, apresentam teores de sal diferentes, o que acaba diminuindo a atividade de água do bonito desidratado osmoticamente e defumado $\left(\mathrm{a}_{\mathrm{w}}=0,971\right)$, em relação ao filé fresco $\left(\mathrm{a}_{\mathrm{w}}=0,987\right)$. Pode-se dizer, ainda, que se tem um produto a alta umidade, uma vez que o processo de desidratação osmótica a que foram submetidos os filés de bonito pode ser considerado brando, reduzindo pouco a umidade do produto. As amostras caracterizadas quanto à dessorção, neste trabalho, são as que têm suas características apresentadas na segunda coluna; os resultados desta tabela se referem a uma média de 10 amostras.

Tabela 1. Caracterização fisico-química do filé de bonito fresco e desidratado osmoticamente e defumado

\begin{tabular}{lcc}
\hline Propriedade & Bonito fresco & Salgado e defumado \\
Umidade (\%) & $72,94 \pm 1,13$ & $71,00 \pm 1,37$ \\
Proteína (\%) & $16,94 \pm 0,67$ & $18,15 \pm 1,67$ \\
Gordura (\%) & $6,95 \pm 1,25$ & $7,44 \pm 0,58$ \\
Cinzas (\%) & $1,39 \pm 0,19$ & $4,05 \pm 0,09$ \\
NaCl (\%) & $0,075 \pm 0,01$ & $2,90 \pm 0,35$ \\
Atividade de água $\left(\mathrm{a}_{\mathrm{w}}\right)$ & $0,987 \pm 0,001$ & $0,971 \pm 0,002$ \\
\hline
\end{tabular}

Na Tabela 2 se encontram os dados que representam os parâmetros dos modelos testados e ajustados para cada uma das temperaturas estudadas e os coeficientes de determinação $\left(\mathrm{R}^{2}\right)$ e o valor do erro relativo médio (MRE). De acordo com os resultados, conclui-se que as constantes dos modelos variaram com a temperatura. O modelo de BET linearizado forneceu valores de umidade de monocamada menores que os obtidos por GAB. Os coeficientes de determinação mais próximos da unidade e valores de erro relativo médio me-

\begin{tabular}{|c|c|c|c|c|c|c|}
\hline \multirow[t]{2}{*}{ Modelos } & \multirow[t]{2}{*}{ Temp. ( $\left.{ }^{\circ} \mathrm{C}\right)$} & \multicolumn{3}{|c|}{ Parâmetros } & \multirow[t]{2}{*}{$\mathrm{R}^{2}$} & \multirow[t]{2}{*}{ MRE (\%) } \\
\hline & & $X_{m}$ & & $\mathrm{C}_{\mathrm{BET}}$ & & \\
\hline \multirow{4}{*}{$\begin{array}{c}\text { BET } \\
\text { (Linearizado) }\end{array}$} & 5 & 0,110 & & $87.360,29$ & 0,945 & 13,13 \\
\hline & 25 & 0,110 & & $7.584,76$ & 0,946 & 13,82 \\
\hline & 40 & 0,096 & & 31,15 & 0,984 & 8,39 \\
\hline & 60 & 0,106 & & 4,69 & 0,991 & 14,61 \\
\hline \multirow{5}{*}{$\mathrm{GAB}$} & & $X_{m}$ & C & $\mathrm{K}$ & & \\
\hline & 5 & 0,176 & 6,718 & 0,942 & 0,998 & 5,68 \\
\hline & 25 & 0,173 & 6,367 & 0,922 & 0,992 & 11,75 \\
\hline & 40 & 0,119 & 6,377 & 0,968 & 0,991 & 11,18 \\
\hline & 60 & 0,090 & 13,570 & 1,020 & 0,993 & 11,02 \\
\hline \multirow{5}{*}{ Henderson } & & $\mathrm{n}$ & & C & & \\
\hline & 5 & 0,815 & & 0,007 & 0,989 & 16,01 \\
\hline & 25 & 0,935 & & 0,008 & 0,984 & 13,23 \\
\hline & 40 & 0,784 & & 0,008 & 0,979 & 19,73 \\
\hline & 60 & 0,654 & & 0,008 & 0,971 & 22,83 \\
\hline \multirow{5}{*}{$\begin{array}{c}\text { Oswin } \\
\text { (Modificado) }\end{array}$} & & A & B & C & & \\
\hline & 5 & $\begin{array}{l}-297,29 \\
\end{array}$ & 59,516 & 0,609 & 0,989 & 15,18 \\
\hline & 25 & $-165,13$ & 33,081 & 0,586 & 0,984 & 11,91 \\
\hline & 40 & 874,650 & $-21,86$ & 0,693 & 0,979 & 13,43 \\
\hline & 60 & 10136,59 & $-168,94$ & 0,830 & 0,971 & 17,00 \\
\hline
\end{tabular}
nores, sugerem que o modelo de GAB descreveu com maior precisão as isotermas de dessorção para atividade de água de 0,112 a 0,893 , com valores de coeficientes de determinação variando de 0,991 a 0,998, observando-se os menores valores de MRE entre os dados experimentais e os preditos.

Tabela 2. Parâmetros de ajuste das isotermas de dessorção de filé de bonito para os diferentes modelos matemáticos

$\mathrm{X}_{\mathrm{m}}$ - umidade de monocamada; $\mathrm{A}, \mathrm{B}, \mathrm{C}, \mathrm{K}$ - parâmetros de ajuste; $\mathrm{R}^{2}$ - coeficiente de determinação; MRE - erro relativo médio 
A umidade de monocamada para os dois modelos, decresce com o aumento de temperatura, como usualmente acontece com outros produtos alimentícios. Pelos coeficientes de determinação, poder-se-ia dizer que todos os modelos apresentaram bom ajuste, porém os altos erros relativos médios observados para os modelos de Henderson e Oswin modificado, permitem indicar BET modificado e GAB como os de melhor ajuste.

Conclui-se então, pelos valores da Tabela 2, que o modelo de GAB representa satisfatoriamente as isotermas de dessorção de filé de bonito salgado e defumado, por ter sido a equação de ajuste que mostrou a melhor combinação entre coeficientes de determinação $\left(\mathrm{R}^{2}\right)$ e os menores valores de erro relativo médio (MRE), para os quatro modelos aqui avaliados. Os valores experimentais e ajustados das isotermas de dessorção de $5,25,40$ e $60{ }^{\circ} \mathrm{C}$, são mostrados na Figura 1, e se pode constatar que as isotermas seguem uma curva do tipo II (Labuza \& Ball, 2000).

Os valores da umidade de monocamada $\left(\mathrm{X}_{\mathrm{m}}\right)$ obtidos com a equação de BET e/ou GAB, estão de acordo e próximos a valores publicados: 7,7; 6,1 e 8,6 g por100 g b.s. para bacalhau, salmão e truta nas temperaturas de 30,37 e $5{ }^{\circ} \mathrm{C}$, respectivamente (Iglesias \& Chirife, 1976). O conteúdo de umidade de equilíbrio a baixas umidades relativas (10 a $50 \%$ ), é quase constante apresentando-se como uma linha ligeiramente horizontal mas a tangente desta linha nesta faixa aumenta, quando decresce a temperatura. O comportamento da isoterma nesta faixa de umidade relativa pode ser interpretado como a estabilidade atingida pelo produto. Um aumento da umidade relativa do meio ambiente nesta faixa não afetará a estabilidade do produto, mas há uma mudança na tangente das curvas à direita das isotermas que, como se pode ver exerceum importante papel na estabilidade e armazenamento do produto, a diferentes temperaturas, uma vez que as variações da umidade de equilíbrio são abruptas e aumentam consideravelmente, para valores de umidades relativas acima de $60 \%\left(\mathrm{a}_{\mathrm{w}}=0,6\right)$.

Em geral, quando a temperatura aumenta, o conteúdo de umidade de equilíbrio decresce para um valor de atividade de água; isto pode ser observado na região de $\mathrm{a}_{\mathrm{w}}$ de 0,30 a 0,80 ,

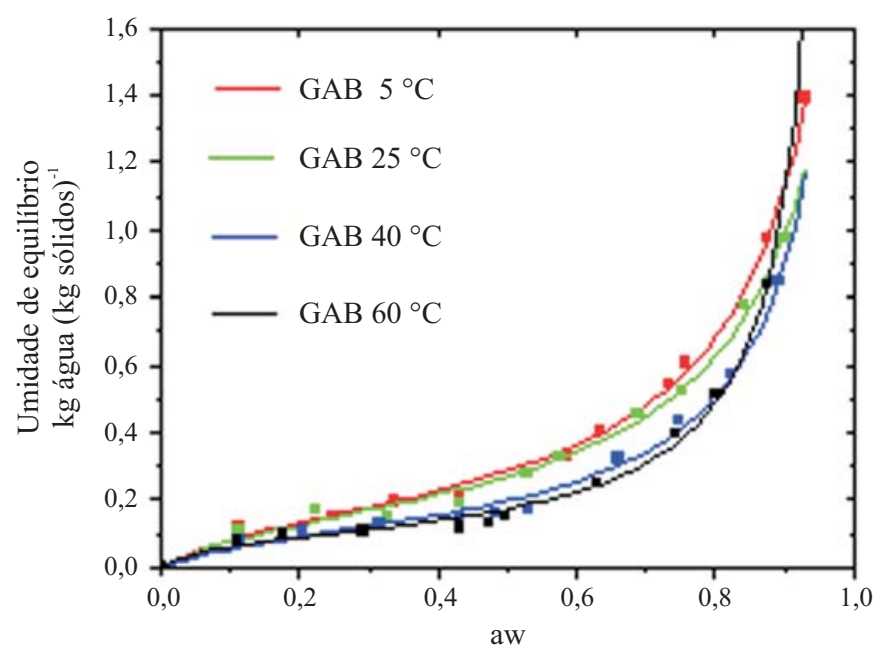

Figura 1. Isotermas de dessorção de filés de bonito (Sarda sarda) na faixa do conteúdo de umidade de equilíbrio de 0,10 a

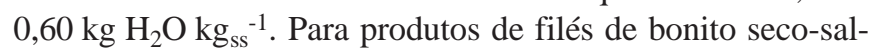
gados e defumados em experimentos prévios, determinou-se que o conteúdo final de umidade deve ser aproximadamente

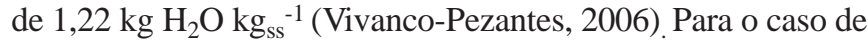
se determinar as condições de armazenamento desse tipo de produto na temperatura de $5{ }^{\circ} \mathrm{C}$, com a equação de $\mathrm{GAB}$ da isoterma de $5^{\circ} \mathrm{C}$, o valor correspondente do ponto de equilíbrio será de $91,8 \%$ de umidade relativa, ou seja, o ar que rodeia o produto deverá ter uma $\mathrm{a}_{\mathrm{w}}=0,918$; este ponto de equilíbrio é importante nos casos de cálculos e especificação e escolha de embalagens (Gal, 1983; Alves et al., 2000).

O calor isostérico de dessorção determinado neste trabalho, possibilita quantificar a demanda energética no processo de secagem do filé de peixe, previamente submetido a uma desidrataçao osmótica (ou salga úmida) e defumado. A curva isostérica de dessorção ( $\operatorname{Ln}\left[\mathrm{a}_{\mathrm{w}}\right]$ em função de 1/T) (Figura 2), para as várias condições de umidade de equilíbrio das amostras, foi obtida com base nos dados das curvas de equilíbrio higroscópico, ajustadas pelo modelo de GAB. O valor do calor isostérico para cada conteúdo de umidade corresponde ao valor do coeficiente de inclinação da reta (Figura 2), multiplicado pela constante universal dos gases ( $\mathrm{R}=0,4618 \mathrm{~kJ} \mathrm{~K}^{-1} \mathrm{~kg} \mathrm{H}_{2} \mathrm{O}^{-1}$ ), conforme propõe a equação de Clausius-Clapeyron.

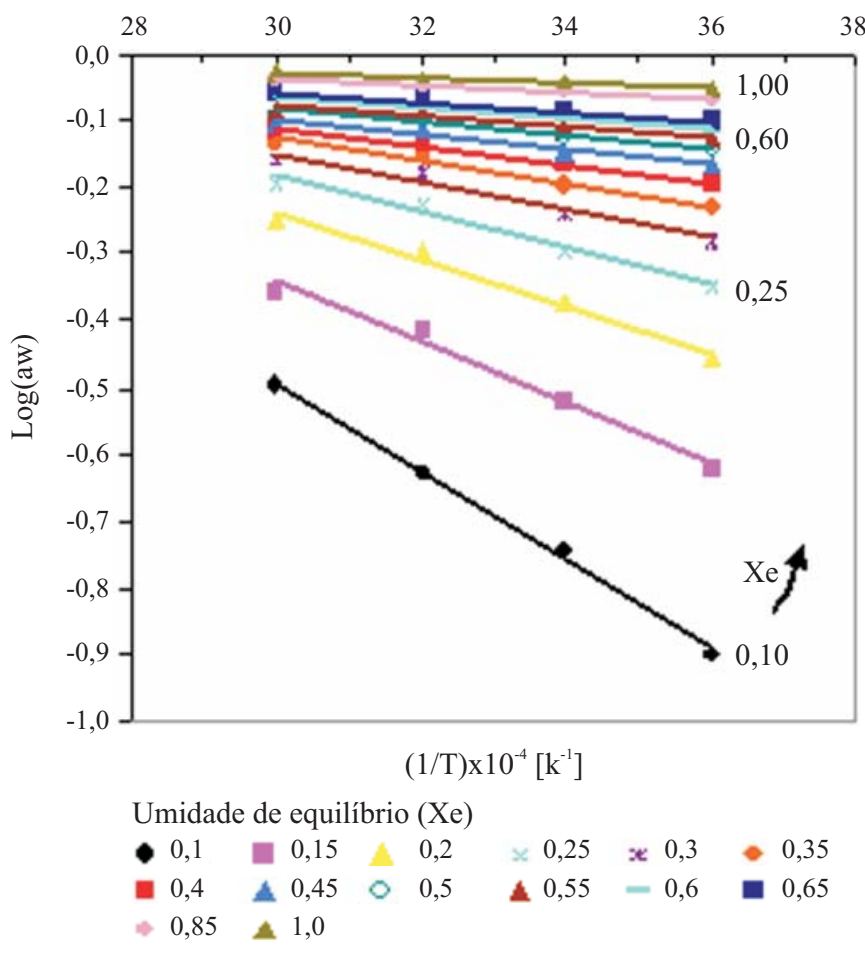

Figura 2. Curvas de $\operatorname{Ln}\left(a_{w}\right)$ versus $1 / T$, baseadas na equação de ClausiusClapeyron, para diferentes níveis de conteúdo de umidade

Dentre esses dados, nota-se decréscimo do calor isostérico $\left(\mathrm{Q}_{\mathrm{ST}}\right)$ com a umidade de equilíbrio, ou seja, quanto menor a umidade do filé do bonito durante a dessorção mais energia é requerida para evaporar a água ligada à estrutura biológica do produto, isto é, a evaporação de água do peixe requer energia para superar o calor de evaporação da 
água pura, sendo baixa a altas umidades e aumentando a baixas umidades (variando, por exemplo: de $32,69 \mathrm{~kJ} \mathrm{~kg}^{-1}$ a $268,11 \mathrm{~kJ} \mathrm{~kg}^{-1}$, para $0,7 \mathrm{~kg} \mathrm{H}_{2} 0 \mathrm{~kg}^{-1} \mathrm{~s}$ e $0,10 \mathrm{~kg} \mathrm{H}_{2} 0 \mathrm{~kg}^{-1} \mathrm{~s}^{-1}$, respectivamente). Resultados e tendências semelhantes foram encontrados por Sablani et al. (2001), ao estudarem isotermas de sorção e o calor isostérico de filés de sardinhas a três temperaturas e para carne salgada, por Igbeka \& Blaisdell (1982).

A Figura 3 mostra a quantidade de energia que deve ser retirada do produto durante a dessorção (secagem), em função de seu conteúdo de umidade. Tem-se que esta energia diminui rapidamente até um conteúdo de umidade de

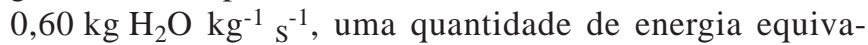
lente a $42 \mathrm{~kJ} \mathrm{~kg}^{-1} \mathrm{H}_{2} \mathrm{O}$ é necessária para evaporar a água, até um valor aproximado de umidade de equilíbrio de $0,60 \mathrm{~kg} \mathrm{H}_{2} \mathrm{O} \mathrm{kgss}^{-1}$. A medida em que a umidade de equilíbrio diminui o valor do calor isostérico aumenta, haja vista que nesse ponto a capacidade de retenção de água do alimento começa a aumentar devido a uma concentração cada vez maior de gorduras, proteínas e sais de cloreto de sódio (na fase aquosa) do alimento. A camada monomolecular fica mais fortemente ligada aos grupos polares dessas substâncias, produzida por um aumento das ligações de hidrogênio das moléculas de água que formam, progressivamente, estruturas ordenadas e rígidas nas quais se precisaria de uma quantidade cada vez maior de energia para quebrá-las e poder liberar uma molécula de água na forma de vapor.

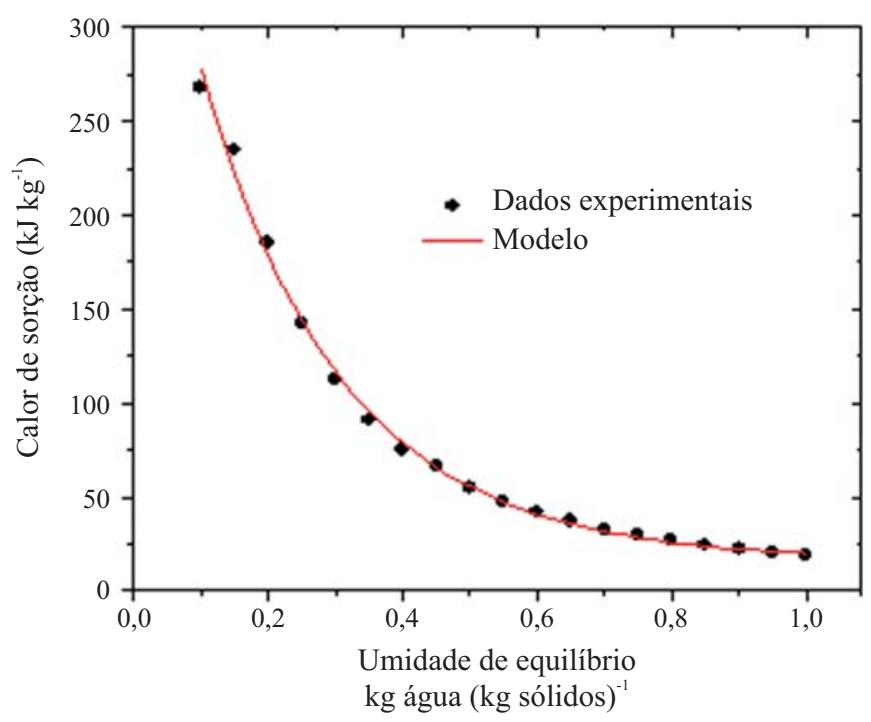

Figura 3. Calor isostérico em função do conteúdo de umidade do pescado salgado e defumado, de acordo com a equação de ajuste proposta neste trabalho

Para o ajuste da curva do calor isostérico $\left(\mathrm{Q}_{\mathrm{st}}\right)$ em função da umidade de equilíbrio $\left(\mathrm{X}_{\mathrm{e}}\right)$ durante a dessorção, propõsse um modelo empírico de três parâmetros que está representado através da Eq. 8, de acordo com discussão apresentada por Vivanco Pezantes (2006).

$$
\mathrm{Q}_{\mathrm{ST}}=\mathrm{A}+\mathrm{B} \cdot \exp \left(-\mathrm{X}_{\mathrm{e}} / \mathrm{C}\right)
$$

Os parâmetros calculados para este ajuste, foram: $A=16,254, B=420,015$ e $C=0,210$, com coeficiente de determinação de 0,996 .

\section{CONCLUSÕES}

1. As isotermas de dessorção de bonito osmoticamente desidratado e defumado apresentaram curva sigmoidal de tipo II e foram bem descritas pela equação de GAB.

2. O conteúdo de umidade da monocamada decresceu com o aumento da temperatura.

3. O calor isostérico de sorção diminuiu com o aumento do conteúdo de umidade.

4. A equação proposta para descrever o calor isostérico em função da umidade de equilíbrio, apresentou um bom ajuste aos dados experimentais.

\section{AGRADECIMENTOS}

Os autores agradecem ao suporte financeiro do Fundo de Amparo à Pesquisa do Estado de São Paulo - FAPESP, Processos No 01/07956-5 e N$^{\circ} 01 / 13809-5$.

\section{LITERATURA CITADA}

Alves, R. M. V; Milanez, C. R; Padula, M. Embalagens alternativas para café solúvel Ciência e Tecnologia de Alimentos, v.20, n.2, p.204-211, 2000.

Anderson, R. B. Modifications of the Brunauer, Emmett and Teller equation. Journal of American Chemistry Society, v.68, p.686-691, 1946.

AOAC - Association of Official Analytical Chemists. Official methods of analysis, 16.ed. Galthersburg: Association of Official Analytical Chemists, 1997. 2v. 40p.

Ariahu, C. C.; Kaze, S. A.; Achem, C. D. Moisture sorption characteristics of tropical fresh water crayfish (Procambarus clarkii). Journal of Food Engineering,v.75, p.355-363, 2006.

Brunauer, S.; Emmett, P. H.; Teller, E. Adsorption of gases in multimolecular layers. Journal of American Chemistry Society, v.60, p.309-319, 1938.

Chen, C. Obtaining the isosteric sorption heat directly by sorption isotherm equations Journal of Food Engineering, v.74, p.178-185, 2006.

Chiralt, A.; Fito, P.; Barat, J. M.; Andres, A.; Gonzales-Martinez, C.; Escriche, I.; Camacho, M. M. Use of vacuum impregnation in food salting process. Journal of Food Engineering, v.40, p.141-151, 2001.

Doe P. E. Fish drying \& smoking: Production and quality. 1.ed. Lancaster: Editorial Technomic, 1998. 250p.

Gal, S. The need for, and practical applications of sorption data. In: Jowitt, R.;. Escher, F.; Hallstrom, B.; Meffert, H.F. T.; Spiess, W.E.L; Vos, G. (ed.) Physical properties of foods, London: Applied Science Publishers, 1983. cap.1, p.13-25. 
Greenspan, L. Humidity fixed points of binary satured aqueous solutions. Journal of Research of the National Bureau of Standards-A. Physics and Chemistry, v.81A, n.1, p.89-96, 1977.

Henderson, S. M. A basic concept of equilibrium moisture. Transactions of the American Society of Agricultural Engineers, v.33, p.9-32, 1952.

Igbeka, J. C.; Blaisdell, J. L. Moisture isotherms of a processed meat product-Bologna. Food Technology, v.17, p.37-46, 1982.

Iglesias H. A.; Chirife J. Isosteric heat of water vapor sorption on dehydrated foods. Part I. Analysis of the differential heat curves. Lebensmittel-Wissenschaft und Technologie, v.9, p.116-122, 1976.

Keachou, N; Maalej, M. Desorption isotherm of imported banana: application of the GAB theory, Drying Technology, v.17, n.6, p.1201-1213, 1999.

Kitic, D.; Jardim, D.; Favetto, G.; Resnik, S.; Chirife, J. Theoretical prediction of the water activity of satured salt sodium solutions at various temperatures. Journal of Food Science, v.51, n.4, p.1037-1041, 1986.

Labuza, T. P.; Ball, L. N.; Moisture sorption-practical aspects of isotherm measurement and use. $2^{\text {nd }}$ ed., Egan:American Association of Cereal Chemist, 2000. 122p.

Labuza,T. P.; Kaane, A.; Chen, J. Y. Effect of temperature on moisture sorption isotherms and water activity shift of two dehydrated foods Journal of Food Science, v.50, p.15-24, 1985.
Molina-Filho, L.; Pedro, M. A. M.; Telis-Romero, J.; Barboza, R. H. R. Influência da temperatura e da concentração de cloreto de sódio $(\mathrm{NaCl})$ nas isotermas de sorção da carne de tambaqui (Colossoma macroparum). Ciência e Tecnologia de Alimentos, v.26, n.2, p.453-458, 2006.

Rucklod, S.; Isengard, H. D.; Hanss, J.; Grobecker, K. H. The energy of interaction between water and surfaces of biological reference materials Food Chemistry, v.82, p.51-59, 2003.

Sabadini, E.; Carvalho, C. B; Sobral, P.; Hubinger, M. Mass transfer and diffusion coefficient determination in wet and dry salting of meat pieces, Drying Technology, v.16 n.9/10, p.2095-2115, 1998.

Sablani, S. S.; Myhara, R. M.; Mahgoub, Z. H.; Al-Attabi., M. M.; Al-Mugheir Y. Water sorption isotherms of freeze dried fish sardines. Drying Technology, v.9, n.3/4, p.673-680, 2001.

Saravacos, G. D.; Tsiourvas, D. A.; Tsami, E. Effect of temperature on water adsorption isotherms of sultana raisins, Journal of Food Science, v.51, n.2, p.381-387, 1986.

Veltchev, Z. N.; Menkov, N. D. Desorption isotherms of apples at several temperatures. Drying Technology, v.18, n.4/5, p.1127-1137, 2000.

Vidal, D. Estudio de la maduración artificial y deshidratación de dátiles españoles. Valencia: UPV, 1983, 158p. Tese Doutorado

Vivanco-Pezantes, D. Estudo das operações combinadas da desidratação osmótica a vácuo, defumação líquida e secagem em filés de bonito (Sarda sarda). Campinas: UNICAMP, 2006, 223p. Tese Doutorado 